\title{
Development and implementation of a dynamic PV emulator with HMI interface for high power inverters
}

\author{
Heredero-Peris D. ${ }^{1}$, Capó-Lliteras M. ${ }^{1}$, Miguel-Espinar C. ${ }^{1}$, Lledó-Ponsati T. ${ }^{2}$, Montesinos-Miracle D. ${ }^{1}$ \\ ${ }^{1}$ Centre d'Innovació Tecnològica en Convertidors Estàtics i Accionaments (CITCEA-UPC) \\ Departament d'Enginyeria Elèctrica, Universitat Politècnica de Catalunya \\ ETS d'Enginyeria Industrial de Barcelona \\ Av. Diagonal 647, 08028 Barcelona, Spain \\ Phone: +34 934016727 \\ Email: daniel.heredero@citcea.upc.edu,macia.capo@citcea.upc.edu, \\ carlos.miguel@citcea.upc.edu, montesinos@ citcea.upc.edu \\ URL:http://www.citcea.upc.edu/ \\ ${ }^{2}$ TeknoCEA, Tecnologies de control de l'electricitat i automatització S.L. \\ Barcelona, Spain \\ Phone:+34 669446622 \\ Email:tomas.lledo@teknocea.cat \\ URL:http://www.teknocea.cat/
}

\section{Acknowledgments}

The authors would like to thank SMA for providing the PV commercial inverter to test the PV emulator and to analyse MPPT of inverter.

\section{Keywords}

$<<$ Photovoltaic $>>,<<$ Voltage Source Converter (VSC) $>>,<<$ Test bench $>>,<<$ Microgrid $>>$, $<<$ DSP $>>$

\begin{abstract}
In this paper a $10 \mathrm{kVA}$ photovoltaic emulator is presented. The system implemented generates photovoltaic V-I characteristic curves to analyse and test maximum power point tracking algorithms of real photovoltaic inverters according to the standard EN50530. The power converter used includes a threephase ac-dc active rectifier. The power stage selected allows to minimize the harmonic current content and permits to be closed to the unity power factor with a fast response. The emulator is controlled by a digital signal processor that communicates with a human-machine-interface. It allows to emulate static and dynamic responses. Furthermore, the interface is also capable of simulating weather evolution and situations under shaded conditions.
\end{abstract}

\section{Introduction}

Nowadays, it is difficult to imagine energy projects where renewable energies are not involved. In this direction, it is important to improve renewable generator technologies and also to develop systems that allow their integration to an utility. Solar and aeolian energy are highly used because sun and wind are free-clean available resources. The tendency toward micro-grids is rising in urban regions in order to improve efficiency and self-managing of the energy. Space requirement and visual impact are important items to take into account. Thus, PV (Photovoltaic) alternatives are a better alternative to be installed. In this sense, developing tools that allow to test commercial systems such as PV inverters and prototypes that use MPPT (Maximum Power Point Tracking) algorithms are required. It is interesting to be able to compare different MPPT techniques under repeatable weather and load conditions. For these reasons, PV emulators that can emulate weather and load conditions including weather forecast are necessary. 
The goal is not be depended on real sunlight. There are two kinds of the principles for PV emulators: controlled incandescent lights and programmable power sources. The main advantages and drawbacks of them are summarized in Table I.

Table I: Comparison of conventional PV emulators.

\begin{tabular}{|c|c|c|}
\hline & Controlled incandescent lights & Programmable power sources \\
\hline$\checkmark$ & Easy sunlight regulation & Easy programming \\
\hline$\checkmark$ & Parcial shading recreation & Several types \\
\hline$\checkmark$ & Real PV element (very high dynamic) & Static and dynamic tests \\
\hline X & Low performance & Expensive \\
\hline X & Low power ranges & Few equipment for high powers \\
\hline X & Need of a real PV element & Own software \\
\hline X & Space requirement & Few manufacturers \\
\hline
\end{tabular}

Each PV generator is defined from non-linear characteristic voltage-current curves that strongly depends on temperature and irradiation. As is shown in Figure 1, $\mathrm{V}_{O C}$ (open circuit voltage) depends strongly on temperature and weakly on irradiation. However, $\mathrm{I}_{S C}$ (short circuit current) depends weakly on temperature and hardily on irradiation.
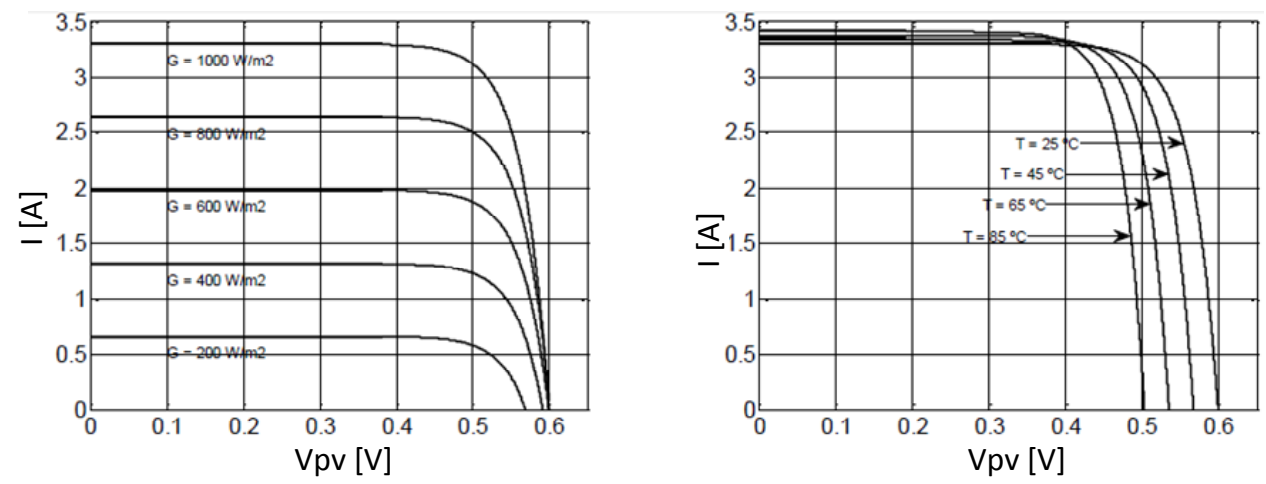

Figure 1: PV cell characteristic curves under irradiation and temperature effect.

On the way a MPPT algorithm is implemented depends the MPP accuracy. When an array is partially shaded or under some bad weather conditions, MPP could not be achieved. Some MPPT algorithms [1],[2] remain in the closest maximum to $\mathrm{V}_{O C}$, regardless if it is the global maximum or not. For these reasons, several times it is wasted large amount of energy. Figure 2 shows an example of a three-serial PV string shaded curve where global maximum does not match with the closest maximum to $\mathrm{V}_{O C}$.

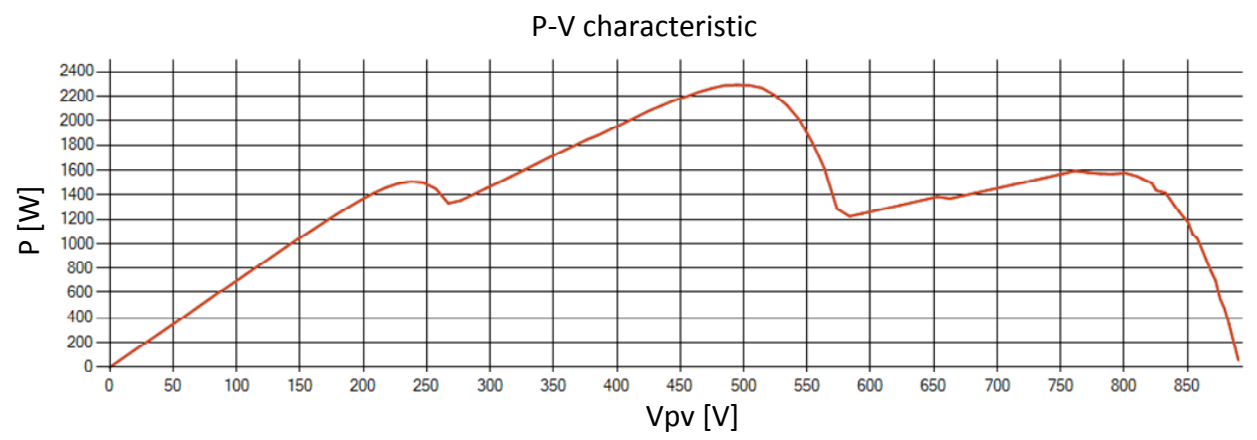

Figure 2: PV string shaded.

On one hand, some PV emulators are implemented by non-controllable diode rectifiers with a buck 
converter [3],[4]. The diode rectifier stage does not allow to control power factor and harmonic current consumption. Furthermore, the buck converter cannot raise voltage and it limits its application fields.

On the other hand, most PV emulators have no HMI (Human Machine Interface). If they have one, a licence program to run them is usually required [5],[6].

This paper presents a $10 \mathrm{kVA}$ PV emulator based on three-phase active rectifier with a third order coupling filter. This filter is constituted by LCL structure and allows to reduce the output voltage and current ripple with reduced space needs, allowing to operate with a nearly constant output. Furthermore, PC HMI has been programmed using Visual $\mathrm{C}++$ to achieve a multi-platform free application that communicates with DSP (Digital Signal Processor) with USB. Figure 3 shows general diagram of the platform.

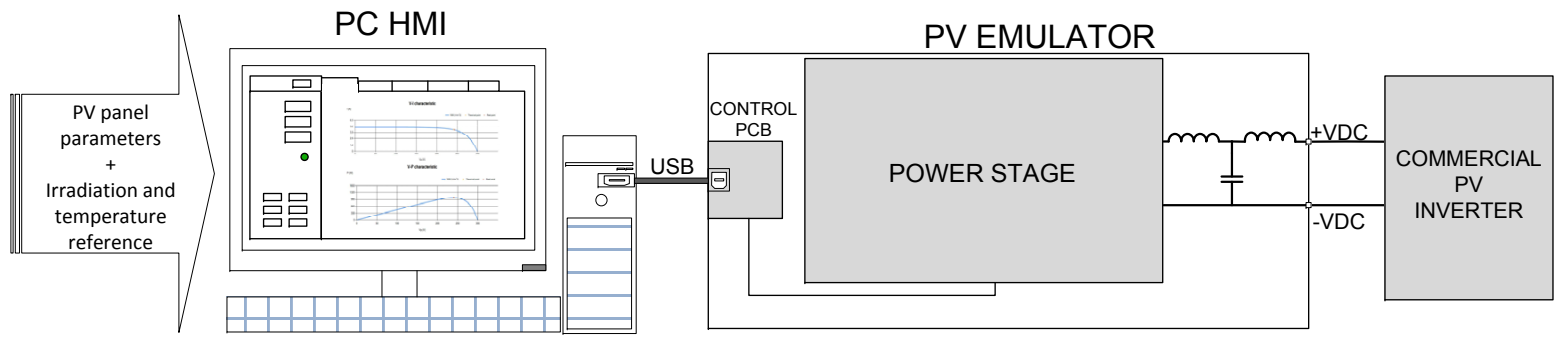

Figure 3: Hardware architecture for PV emulator platform implemented.

\section{System description and control strategy}

There are two major circuit sections: control and power stage circuits, and PC HMI. Power stage is constituted by an isolation transformer with an inductive input coupling filter and a three-phase active rectifier with an LCL output filter. The PC HMI has been programmed using Visual C++ to obtain a complete application that fulfils EN50530.

The active rectifier does not allow to operate through the overall PV curve because active rectifier operates as a boost converter. The minimum theoretical synthesizable output voltage is the rectified input voltage. However, PV inverters usually operate on region between $\mathrm{V}_{O C}$ and $\mathrm{V}_{M P P}$. Moreover this configuration has better efficiency and the system can be smaller and cheaper than others configurations with more power stages. Figure 4 shows this configuration.

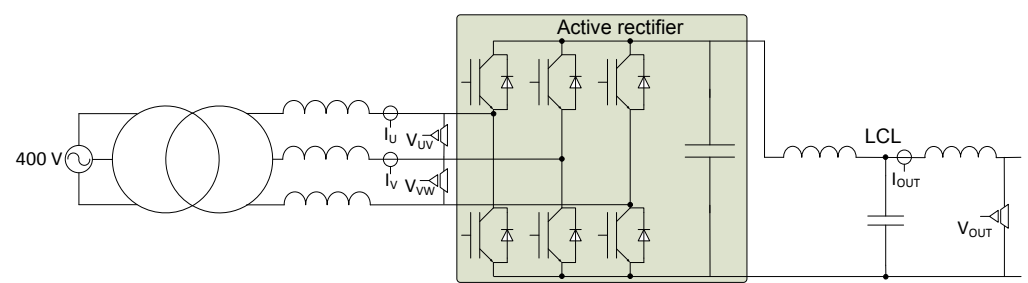

Figure 4: PV hardware configuration proposed.

Some desired functional features for the PV emulator operation are for example low space requirements, flexibility to load or download curves, representation of theoretical and real operation points, test standardization, among others.

\section{Active rectifier modelling}

The active rectifier stage implemented is constituted by a three-leg structure. In this way, the use of Park transformation facilitates control tasks in order to operate in a synchronous reference. The desired control system has to act by means of a synchronous inner current loop. The matrix equation that describes the dynamic behaviour of three-phase inductances between two nodes is

$$
\frac{d}{d t}\left(\begin{array}{c}
I_{A} \\
I_{B} \\
I_{C}
\end{array}\right)=-\left(\begin{array}{ccc}
\frac{R}{L} & 0 & 0 \\
0 & \frac{R}{L} & 0 \\
0 & 0 & \frac{R}{L}
\end{array}\right)\left(\begin{array}{c}
I_{A} \\
I_{B} \\
I_{C}
\end{array}\right)+\left(\begin{array}{ccc}
\frac{1}{L} & 0 & 0 \\
0 & \frac{1}{L} & 0 \\
0 & 0 & \frac{1}{L}
\end{array}\right)\left(\begin{array}{c}
V_{A}-V_{U} \\
V_{B}-V_{V} \\
V_{C}-V_{W}
\end{array}\right)
$$


being $\left(\mathrm{V}_{U}, \mathrm{~V}_{V}, \mathrm{~V}_{W}\right)$ the simple grid voltages, $\left(\mathrm{I}_{A}, \mathrm{I}_{B}, \mathrm{I}_{C}\right)$ the line currents and $\left(\mathrm{V}_{A}, \mathrm{~V}_{B}, \mathrm{~V}_{C}\right)$ the active rectifier output voltages. In this way, using the Park transformation that normalizes the variables maintaining the power and applying it to (1) can be obtained

$$
\frac{d}{d t}\left(\begin{array}{c}
I_{d} \\
I_{q}
\end{array}\right)=-\left(\begin{array}{cc}
\frac{R}{L} & -\omega \\
\omega & \frac{R}{L}
\end{array}\right)\left(\begin{array}{c}
I_{d} \\
I_{q}
\end{array}\right)+\left(\begin{array}{cc}
\frac{1}{L} & 0 \\
0 & \frac{1}{L}
\end{array}\right)\left(\begin{array}{c}
V_{c d}-V_{g d} \\
V_{c q}-V_{g q}
\end{array}\right)
$$

taking into account that in a three-phase system without accessible neutral point there is no homopolar component and considering that the $\mathrm{V}_{g q}$ is zero thanks to PLL. Thus, $\left(\mathrm{I}_{d}, \mathrm{I}_{q}\right)$ are the $d$ - $q$ current components, $\mathrm{V}_{c d}, \mathrm{~V}_{c q}$ are the simple $d-q$ voltage components of the converter and, finally, $\left(\mathrm{V}_{g d}, \mathrm{~V}_{g q}\right)$ corresponds to the grid $d-q$ components. The scheme described in Figure 5(a) can be obtained, where $\left(m_{1}, m_{2}\right)$ are the input signals, $\left(y_{1}, y_{2}\right)$ the output response, $a_{x x}$ the direct transmittances and $a_{x y}$ the coupling transmittances.

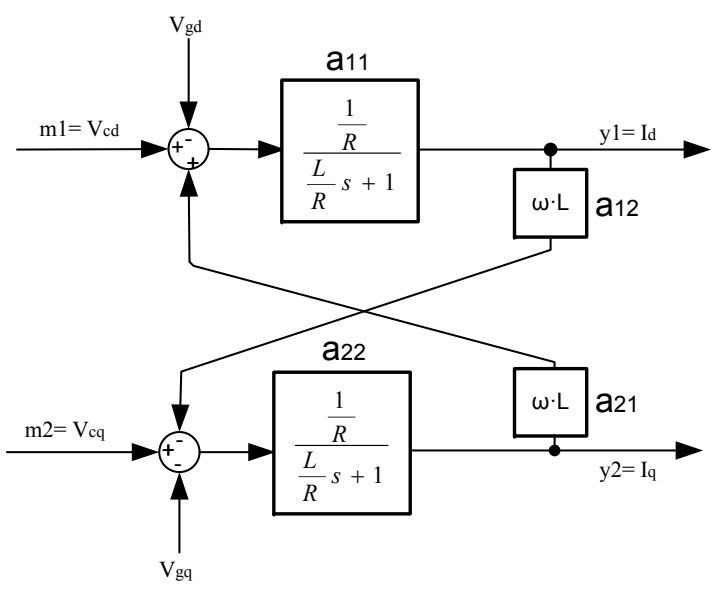

(a) Coupling system describing the open loop diagram.

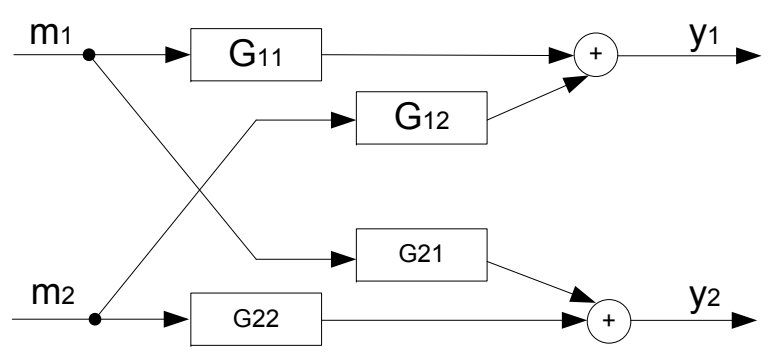

(b) Desired input/output coupling scheme for RGA application.

Figure 5: Control block diagrams.

It is possible to observe that this system is coupled. To control a coupled system there are three mainly options: decoupling the system, multi-variable control or study the importance of the coupling and try to neglect it if possible. In this case, the last option is chosen. For this reason, the coupling relationships are evaluated using the RGA (Relative Gain Array) strategy, also known as Bristol method [7]-[10]. Inputs of the system $\mathrm{V}_{g d}, \mathrm{~V}_{g q}$ are considered null because a feed-forward in the inner controller is implemented. For the calculations of the RGA, it is interesting that the system presents the structure of interaction that is shown in Figure 5(b), where $G_{x x}$ and $G_{x y}$ are the direct and coupling transmittances, respectively. Thus, for Figure 5(b) $\mathbf{y}(\mathbf{s})=\mathbf{G}(\mathbf{s}) \mathbf{m}(\mathbf{s})$. The system to control has an output-input coupling instead of the desired input-output coupling of Figure 5(b). Considering the relations from Figure 5(a), $y_{1}$ and $y_{2}$ can be obtained as

$$
\left\{\begin{array}{l}
y_{1}=\left(m_{1}+y_{2} a_{21}\right) a_{11}=\left(m_{1}+\left(\left(m_{2}-y_{1} a_{12}\right) a_{22}\right) a_{21}\right) a_{11} \\
y_{2}=\left(m_{2}-y_{1} a_{12}\right) a_{22}=\left(m_{2}-\left(\left(m_{1}+y_{2} a_{21}\right) a_{11}\right) a_{12}\right) a_{22}
\end{array}\right.
$$

and rearranging some terms

$$
\left\{\begin{array}{c}
y_{1}\left(1+\prod^{\forall i, j}\left[a_{i j}\right]\right)=m_{1} a_{11}+m_{2} a_{21} a_{11} a_{22} \\
y_{2}\left(1+\prod^{\forall i, j}\left[a_{i j}\right]\right)=m_{2} a_{22}-m_{1} a_{12} a_{11} a_{22}
\end{array}\right.
$$

So, according to Figure 5(b), the desired transmittance matrix $\mathbf{G ( s )}$ is

$$
\mathbf{G}(\mathbf{s})=\left(\begin{array}{ll}
G_{11}(s) & G_{12}(s) \\
G_{21}(s) & G_{22}(s)
\end{array}\right)
$$


constituted by $G_{x x}$ and $G_{x y}$ that, for the proposed current loop analysis, can be deduced that

$$
\left\{\begin{array}{c}
G_{11}(s)=G_{22}(s)=\frac{a_{11}}{\left(1+\Pi^{\forall i, j}\left[a_{i j}\right]\right)}=\frac{L s+R}{(L s+R)^{2}+(\omega L)^{2}} \\
G_{12}(s)=-G_{21}(s)=\frac{a_{11} a_{22} a_{12}}{\left(1+\prod^{\forall i, j}\left[a_{i j}\right]\right)}=\frac{\omega L}{(L s+R)^{2}+(\omega L)^{2}}
\end{array}\right.
$$

because $a_{11}=a_{22}$ and $a_{12}=a_{21}$. For the RGA method it is necessary to follow next three steps:

- Determination of the relative gain array of Bristol. Once the four transfer functions required are obtained $\left(\mathrm{G}_{11}-\mathrm{G}_{12}-\mathrm{G}_{21}-\mathrm{G}_{22}\right)$, it is possible to proceed evaluating $\mathbf{G}(\mathbf{s})$ at steady state as $\mathbf{G}(\mathbf{0})$, obtaing the static gain matrix as

$$
\mathbf{K}=\left(\begin{array}{ll}
k_{11} & k_{12} \\
k_{21} & k_{22}
\end{array}\right)=\left(\begin{array}{cc}
\frac{R}{(R)^{2}+(\omega L)^{2}} & \frac{\omega L}{(R)^{2}+(\omega L)^{2}} \\
\frac{-\omega L}{(R)^{2}+(\omega L)^{2}} & \frac{R}{(R)^{2}+(\omega L)^{2}}
\end{array}\right)
$$

and its transpose inverse is defined as

$$
\mathbf{K}^{-t}=\frac{1}{k_{11} k_{22}-k_{12} k_{21}}\left(\begin{array}{cc}
k_{22} & -k_{21} \\
-k_{12} & k_{11}
\end{array}\right)
$$

Therefore, the $\boldsymbol{\Lambda}$ RGA system is defined as the Hadamard product, element-by-element, of $\mathbf{K} \times \mathbf{K}^{-t}$

$$
\boldsymbol{\Lambda}=\left(\begin{array}{ll}
\lambda_{11} & \lambda_{12} \\
\lambda_{21} & \lambda_{22}
\end{array}\right)
$$

where the relative gain $\lambda_{i j}=\frac{\left.\frac{d y_{i}}{d m_{j}}\right|_{\text {all loopsopen }}}{\frac{d y_{i}}{d m_{j}} \mid \text { alloopsopenexcept toop } m_{j}}$. That means that, for instance, $\lambda_{12}$ represents the relationship of the gain between input $m_{2}$ and output $y_{1}$ with $m_{2}$ constant respect the gain between input $m_{2}$ and output $y_{1}$ with $y_{1}$ constant.

- Choose the best coupling between inputs and outputs. Summarising all the possibilities, if the component $\lambda_{i j}$ of $\boldsymbol{\Lambda}$ is close to one, input ${ }_{j}$ controls independently output $t_{i}$. If the value is close to zero practically there is not interaction between input $_{j}$ and out put $_{i}$. For negative values or really high values, the detailed implications are detailed in [8].

- Check if the pairing is quite significant.

- Achieve independent tuning of the controller if all previous steps are valid. If not, a classical decoupling strategy or multi-variable control has to be performed.

As an example, if a coupling inductance of $1 \mathrm{mH}$ with a parasitic resistance of $1 \Omega$ is considered, the RGA obtained for the $2 \times 2$ system under study is

$$
\mathbf{\Lambda}=\left(\begin{array}{cc}
\frac{k_{11} k_{22}}{k_{11} k_{22}-k_{12} k_{21}} & 1-\lambda_{11} \\
1-\lambda_{11} & \lambda_{11}
\end{array}\right)=\left(\begin{array}{cc}
0.9102 & 0.0898 \\
0.0898 & 0.9102
\end{array}\right)
$$

implying that direct components are strongly linked while coupling terms (secondary diagonal) shows weak interaction. Taking into account all the steps aforementioned, control scheme for the active rectifier stage can be obtained if a weak interaction is probed. Figure 6 shows how the $\mathrm{I}_{B U S}$ reference from a characteristic PV curve table is obtained. This table is obtained applying EN50530 mathematical curve model in HMI and sent to DSP by USB. Other needed values are obtained interpolating. Current reference changes depending on operating output voltage point imposed by the MPPT of PV inverter. Block called Id calculation allows to implement a power balance taking into account the losses in the AC input inductance. So, describing the power balance between the input and the output of the PV emulator as

$$
\begin{aligned}
& P_{D C}=P_{\text {lose }(L R)}+P_{d q}=V_{D C} I_{D C}=3 R_{L} I_{R M S}^{2}+\left(V_{d} I_{d}+V_{q} I_{q}\right)= \\
& 3 R_{L}\left(\frac{I_{d}}{\sqrt{3}}\right)^{2}+\left(V_{d} I_{d}+V_{q} I_{q}\right)=R_{L} I_{d}^{2}+\left(V_{d} I_{d}+V_{q} I_{q}\right)
\end{aligned}
$$

where $P_{D C}$ is the power at the DC-link side, $P_{\text {lose }(L R)}$ the losed power at the AC copuling filter considering $R_{L}$ the parasitic resistance of the inductance in (11). Imposing $I_{q}=0$, the Id reference can be deduced. 


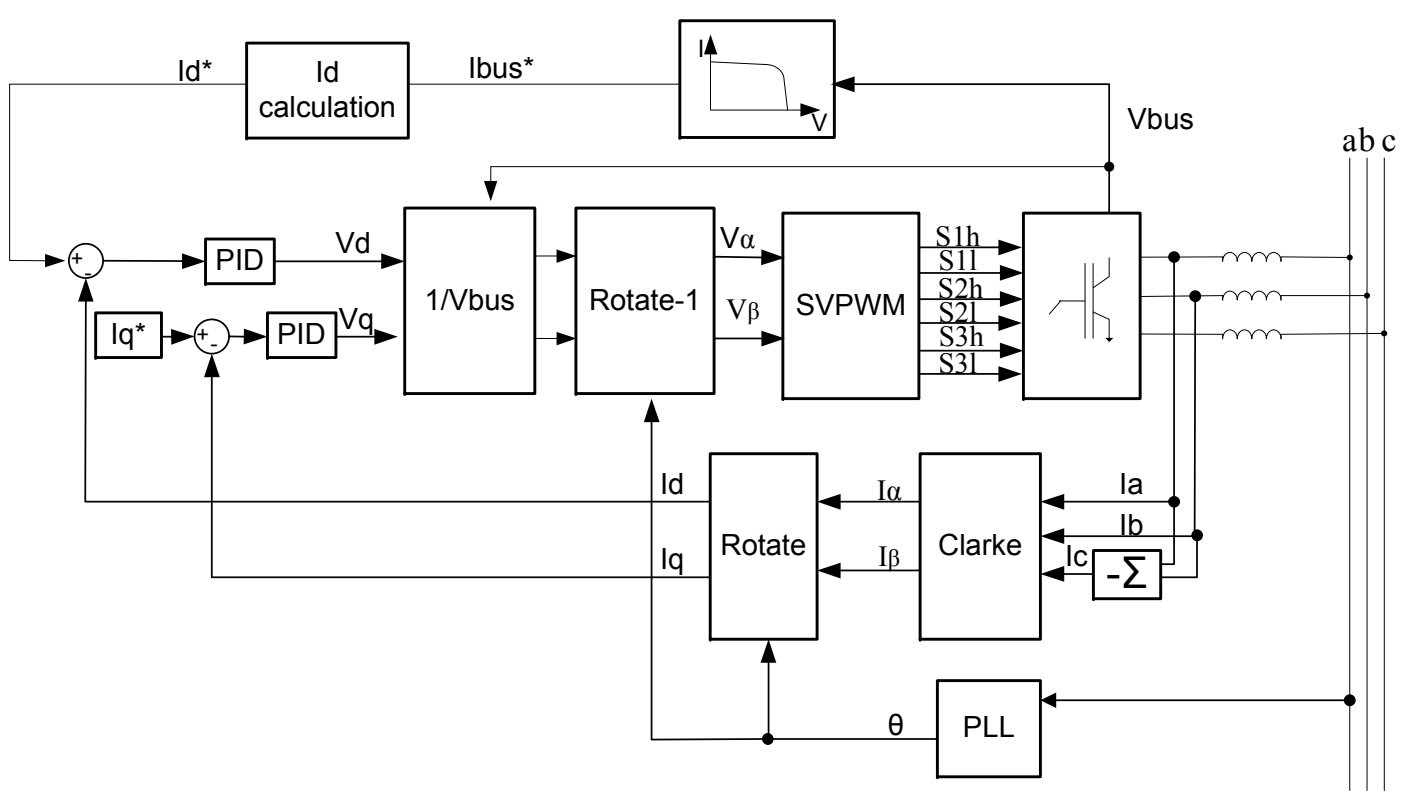

Figure 6: Control scheme for the active rectifier stage.

\section{HMI}

The PC HMI has been developed in Visual Studio C++. The structure of the HMI has to be friendly. For this reason, it only has one window where there is a common region where user can open the communication USB port, introduce common parameters and see the operating status of the converter. Furthermore, there is a pop-up menu that allows to save graphical results with labels that informs of which is the real and theoretical point. The HMI structure is shown in Figure 7.

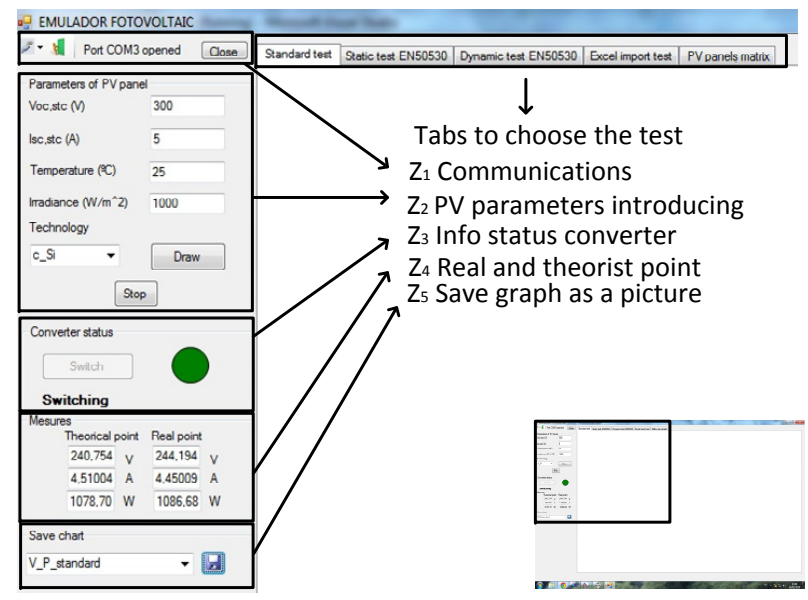

Figure 7: HMI structure.

Also, there is the test region which occupy the main area of the window, where the user can select different proposed tests. There has been considered 5 different tests selectable through different tabs, as can be observed in Figure 8.

\section{\begin{tabular}{|l|l|l|l|l|}
\hline Standard test & Static test EN50530 & Dynamic test EN50530 & Excel import test & PV panels matrix \\
\hline
\end{tabular}}

Figure 8: Tabs of each test that it's capable of executing. 
The implemented tests are:

Standard test: The user introduce panel parameters and send them to the DSP to emulate the desired characteristics. As it is shown in Figure 9(a) it is displayed the V-I and P-V PV characteristic curves, the theoretical and the operating point.

Static test: This test automates the proceeding to calculate the MPPT efficiency in static conditions according to the standard EN50530. The total efficiency is computed as a result of adding all efficiencies, which are calculated in different power rates of the inverter. Overall efficiency is obtained applying to each one the corresponded weight factor. The user only has to introduce the inverter parameters. The results that can be observed includes the characteristic curves, a table where appear each efficiency calculated and the theoretical power versus real power time evolution. This test is shown in Figure 9(b).

Dynamic test: This test automates the proceeding to calculate the MPPT in dynamic irradiation conditions according to the standard EN50530. This standard fixes a pattern to calculate the MPPT inverter efficiency under these conditions. The user can see theoretical versus real power time evolution and the calculated efficiency that can be seen in Figure 9(c).

Excel import test: This test allows to import irradiation and temperature evolution from a .csv file. HMI represents this evolution and the process of the test in a plot. The user can choose the scale of time to make compressed time validations. Furthermore, like others previous tests, the PV characteristics curves are displayed. This test is shown in Figure 9(d).

$P V$ panels matrix: This test allows to emulate a series-parallel panel matrix under different irradiations. The resultant curve has different local maximums as it is shown in Figure 9(e). The aim of this calculation is checking if the inverter under test is capable of operating in the global maximum.

\section{Results}

The converter test platform consists in a three-phase active rectifier with LCL filter to the output and the modulation technique chosen is the SVPWM (Space Vector Pulse Width Modulation) [11]. The general features considered of platform are shown in Table II and in Figure 10 a photograph of the test bench is shown.

Table II: General features of the experimental set-up

\begin{tabular}{|l|l||l|l|}
\hline Parameter & Feature & Component & Values \\
\hline USB & $76800 \mathrm{bps}$ & AC inductance & $1 \mathrm{mH}$ \\
DSP & 32 bits fixed point & AC parasitic resitance & $1 \Omega$ \\
HMI & Visual Studio C++ & Switching frequency & $20 \mathrm{kHz}$ \\
Max. power converter & $10 \mathrm{kVA}$ & Control frequency & $20 \mathrm{kHz}$ \\
Output DC voltage & $0-750 \mathrm{~V}$ & SMA commercial inverter & $2 \mathrm{~kW}$ \\
Maximum DC current & $30 \mathrm{~A}$ & DC bus & $2 \mathrm{mF}$ \\
\hline
\end{tabular}

The results presented in Figure 11 has been obtained connecting a commercial photovoltaic inverters downstream the PV emulator that has provided by SMA. When the commercial inverter detects DC voltage itself, after few time if grid is stable enough, it starts to inject power into the grid according to its MPPT algorithm. Figure 11(a) is a oscilloscope capture that shows the V-I evolution starting from $300 \mathrm{~V}$ open-circuit voltage up to a $240 \mathrm{~V}$ (MPP). Figure 11(b) shows the tab of the HMI, where it is shown how real and theoretical point match at the end.

In Figure 11(a), on bottom left, it can be observed MPPT path of V-I plot. On bottom right, it can be observed MPPT path of V-P plot. Moreover, on top of the Figure 11(a), it is shown time evolution of the voltage, current and power. It can see how voltage (purple) remains on $\mathrm{V}_{O C}$ until inverter starts to inject. Afterwards, voltage decrease and current and power rise up. Finally it is also observable how the inverter applies $5 \mathrm{~V}$ step up and down every 2 seconds to know in which side of the MPP is operating.

\section{Conclusions}

This paper has presented a PV emulator that is constituted by an active rectifier with LCL filter to the output. A non specifically decoupled control has been justified using the Bristol method based on RGA for the active rectifier stage that operates as a current source. Platform has been tested with an HMI that integrates communications by USB between PC and the control board. A flexible battery of tests are supported also considering standard EN50530. Moreover, all these tests have been checked connecting the PV emulator to a SMA commercial inverter that it has become possible to analyse its MPPT algorithm. 


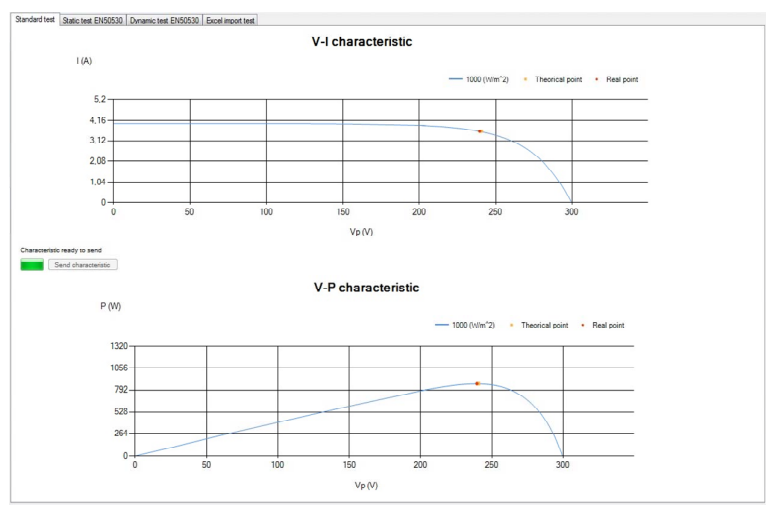

(a) Standard test.

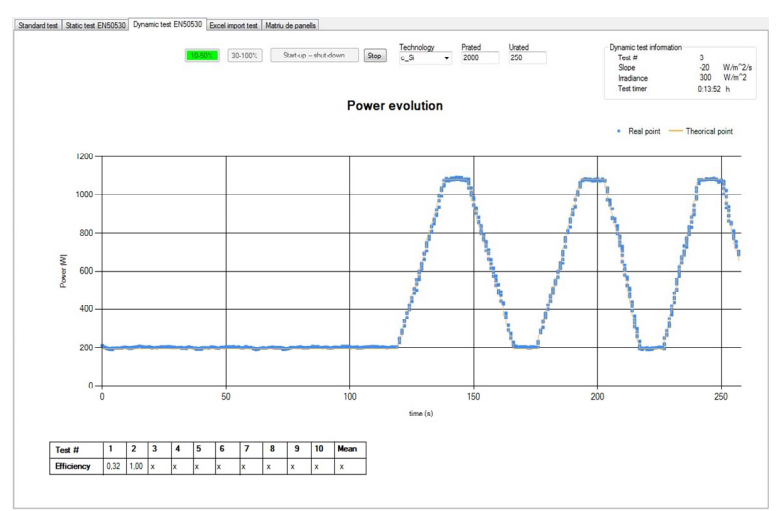

(c) Dynamic test EN50530.

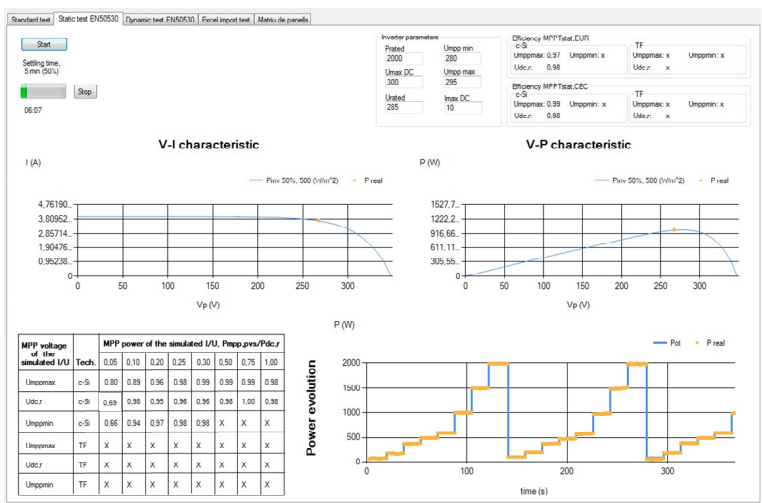

(b) Static test EN50530.

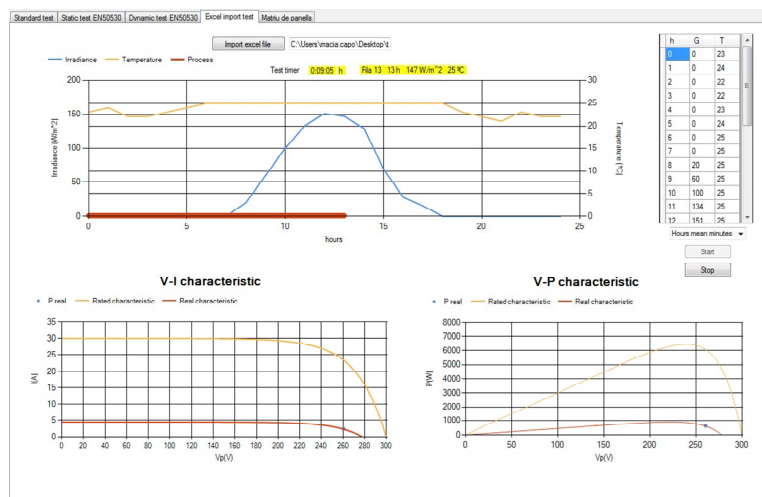

(d) Excel import test.

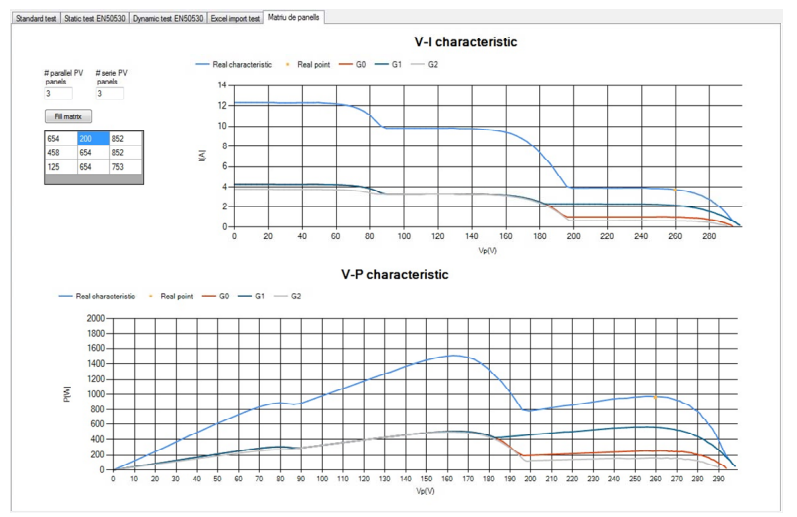

(e) PV panel matrix test.

Figure 9: HMI tabs 


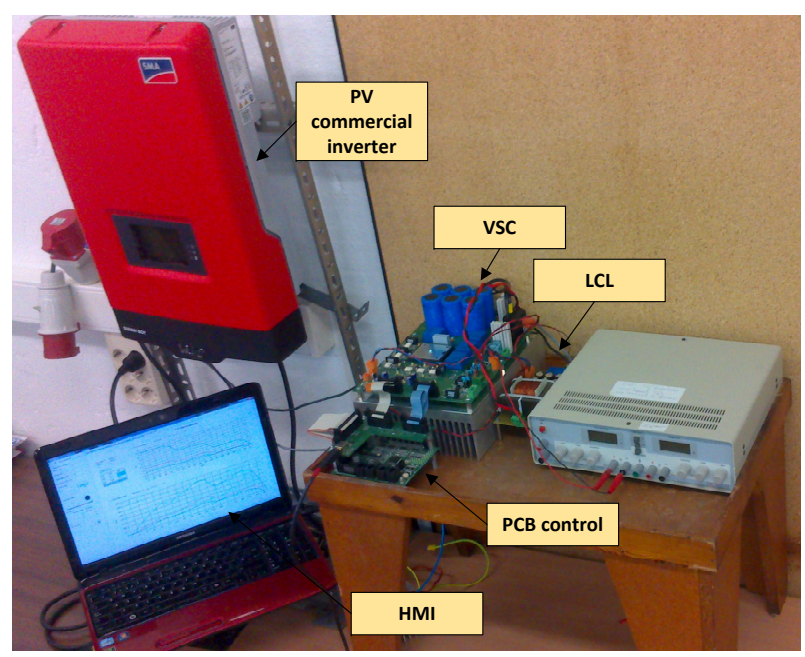

Figure 10: Test bench photograph.

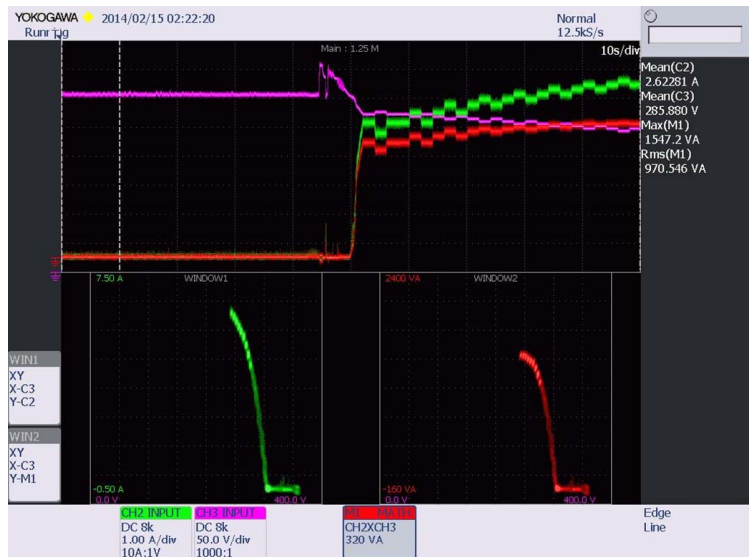

(a) Commercial inverter MPPT path response, oscilloscope capture.

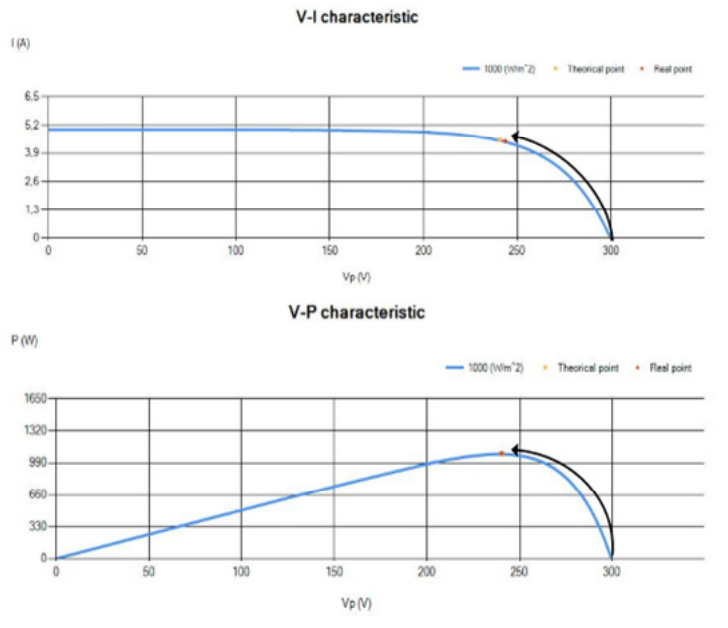

(b) Commercial inverter MPPT path response, HMI capture.

Figure 11: Commercial inverter MPPT path response. 


\section{References}

[1] T. Esram and P. L. Chapman, "Comparison of photovoltaic array maximum power point tracking techniques." IEE Transactions on Energy Coversion, vol. 22 no 2, pp. 439-449, 2007.

[2] W. Xiao, A. Elnosh, V. Khardkidar, and H. Zeineldin, "Overview of maximum power point tracking technologies for photovoltaic power systems"; in IEEE Industrial Electronics Society Conference (IECON), 2011, pp. 3900-3905.

[3] A. Koran, K. Sano, Rae-young Kim, and Hih-Sheng Lai, "Design of a Photovoltaic Simulator with a novel references signal generator and two-stage LC Output", IEE Transaction on Power Electronics, vol. 25, no. 5 pp 1331-1338, 2010.

[4] Ho Lee, Min-Jung Lee, Se-Naa Lee, Hwa-Chun Lee, Hae-Kon Nam, and Sung-Jun Park, "Development of photovoltaic simulator based on DC-DC converter", Telecommunications Energy Conference 31st International, pp. 1-5, 2009.

[5] L. Bun, B. Raison, G. Rostaing, S. Bacha, A. Rumeau, A. Labonne, "Development of a real time photovoltaic simulator in normal and abnormal operations", (IECON) Annual Conference on IEEE Industrial Electronics Society, pp. 867-872, 2011.

[6] Riad Kadri, Jean-Paul Gaubert, Gerard Champenois and Mohamed Mostafai, "Real-time emulator of photovoltaic array in partial shadow conditions based on closed-loop reference model", Scientific Bulletin of the Electrical Engineering Faculty, 2010.

[7] Ajayi T.O., I.S.Ogboh., "Determination of Control Pairing for Higher Order Multivariable Systems by the use of Multi-Ratios", International Journal of Scientific and Engineering Research Volume 3, Issue 3, March 2012.

[8] Wayne Bequette B., "Process Control. Modeling, Designs and Simulation", Prentice Hall PTR, December 26,2002

[9] Wuhua Hu; Wen-Jian Cai; Gaoxi Xiao, "Relative gain array for MIMO processes containing integrators and/or differentiators," Control Automation Robotics \& Vision (ICARCV), 2010 11th International Conference on , vol., no., pp.231,235, 7-10 Dec. 2010

[10] Li-Jun Cai; Erlich, I., "Identification of the Interactions among the Power System Dynamic Voltage Stability Controllers using Relative Gain Array," Power Systems Conference and Exposition, 2006. PSCE '06. 2006 IEEE PES, vol., no., pp.970,977, Oct. 29 2006-Nov. 12006

[11] Shijie Yan; Qun Zhang; Heng Du, "A simplified SVPWM control strategy for PV inverter," Control and Decision Conference (CCDC), 2012 24th Chinese, vol., no., pp.225,229, 23-25 May 2012 